\title{
Excessive positive fluid balance has a negative effect on short-term renal outcomes after kidney transplantation
}

\author{
Jun Gyo Gwon ${ }^{1}$, Myung-Gyu Kim², Cheol Woong Jung ${ }^{1}$, Chang Hun Lee ${ }^{1}$ \\ ${ }^{1}$ Division of Transplantation, Department of Surgery, Korea University Anam Hospital, Seoul, Korea \\ ${ }^{2}$ Division of Nephrology, Department of Internal Medicine, Korea University Anam Hospital, Seoul, Korea
}

Background: Optimized postoperative fluid management is important in maintaining early allograft function after kidney transplantation (KT). However, there is still no clear guidance on how to treat fluid after KT. In this study, we investigated the effect of fluid balance on postoperative allograft function.

Methods: Recipients underwent KT between March 2012 and August 2018 were included and their medical records were reviewed retrospectively. We calculated fluid balance, which is the difference between total input and output during 3 days after KT, and analyzed the change of estimated glomerular filtration rate (eGFR) according to fluid balance.

Results: A total of 178 patients were included, except those with delayed graft function or urine output less than $2,000 \mathrm{~mL}$ on the first day after KT. Among them, 116 received kidneys from living donors and 62 from deceased donors. The total fluid balance up to day 3 was $4,236.9 \pm 2,830.4 \mathrm{~mL}$. Old age, high body mass index (BMI), excessive positive fluid balance of recipient and high final creatinine of donor were significantly associated with low eGFR at 1 week. In addition, age, BMI, and fluid balance predicted 1-month eGFR. In multivariate analysis, an excessive positive fluid balance was an independent factor to predict low 1-week eGFR $(P=0.031)$. In particular, this positive correlation was evident in the living donor KT recipients, suggesting that excessive positive fluid balance at early postoperative period can delay functional recovery of the transplanted kidney, regardless of donor kidney status.

Conclusions: We have demonstrated that excessive positive fluid balance can negatively affect early graft function after KT. Therefore proper fluid management strategies based on volume conditions may provide new therapeutic opportunities to improve early renal outcomes after KT.

Corresponding author: Jun Gyo Gwon

E-mail: doctorgjg@gmail.com

(C) The Korean Society for Transplantation

This is an Open Access article distributed under the terms of the Creative Commons Attribution Non-Commercial License (http://creativecommons.org/licenses/by-nc/4.0/) which permits unrestricted non-commercial use, distribution, and reproduction in any medium, provided the original work is properly cited. 